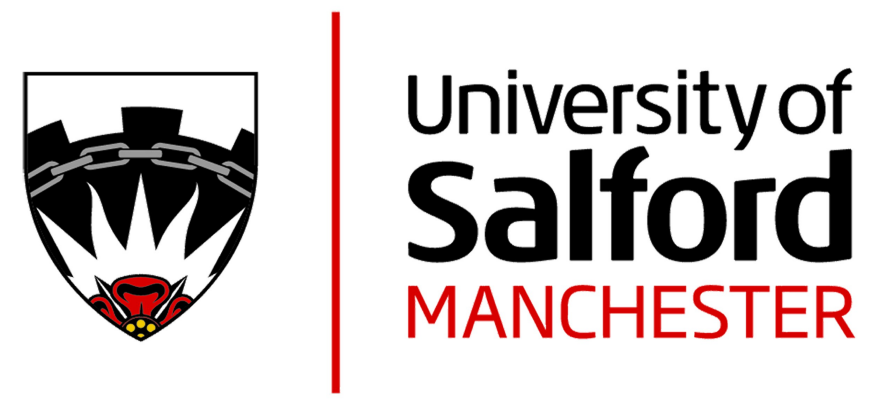

\title{
In-situ sub-structure decoupling of resiliently coupled assemblies
}

Meggitt, J.W.R. and Moorhouse, A.T.

\begin{tabular}{|l|l|}
\hline Title & $\begin{array}{l}\text { In-situ sub-structure decoupling of resiliently coupled } \\
\text { assemblies }\end{array}$ \\
\hline Authors & Meggitt, J.W.R. and Moorhouse, A.T. \\
\hline Type & Article \\
\hline URL & $\begin{array}{l}\text { This version is available at: } \\
\text { http://usir.salford.ac.uk/id/eprint/52926/ }\end{array}$ \\
\hline Published Date & 2019 \\
\hline
\end{tabular}

USIR is a digital collection of the research output of the University of Salford. Where copyright permits, full text material held in the repository is made freely available online and can be read, downloaded and copied for noncommercial private study or research purposes. Please check the manuscript for any further copyright restrictions.

For more information, including our policy and submission procedure, please contact the Repository Team at: usir@salford.ac.uk. 


\title{
In-situ sub-structure decoupling of resiliently coupled assemblies
}

\author{
J.W.R.Meggitt ${ }^{1}$, A.T.Moorhouse ${ }^{1}$ \\ ${ }^{1}$ Acoustics Research Centre, University of Salford, Greater Manchester, M5 4WT
}

\begin{abstract}
The context of this paper is the increasing need for vibro-acoustic simulation across many sectors. A procedure is derived for decoupling the components of resiliently mounted assemblies. An independent characterisation of the components is obtained such that they can be mathematically recombined with other elements to form virtual assemblies or Virtual Acoustic Prototypes. Unlike standard decoupling procedures, the proposed approach does not require the assembly to be physically decoupled at any stage. It is argued that this offers significant advantages in terms of convenience and, importantly, representativeness. The boundary conditions within a physically coupled assembly are realistic by definition, which may not be the case for physically decoupled components. The procedure is validated numerically using a lumped parameter model and demonstrated experimentally through several case studies.
\end{abstract}

Keywords: In-situ, measurement, experimental,free-interface, decoupling, FRF, sub-structure, structural, characterisation

\section{Introduction}

A drive towards leaner engineering has seen the use of physical prototypes become a limiting factor in both the development of new products, and the continual development of existing products. As such, alternative prototyping methods are of interest. For the assessment of vibro-acoustic performance the aptly named 'virtual acoustic prototype' (VAP) offers a convenient alternative [1]. A robust and experimentally focused VAP is built on the foundation of dynamic sub-structuring (DS) [2], where structural components are coupled together mathematically through the application of continuity and equilibrium constraints [3]. DS techniques allow for the convenient interchange and/or modification of components within a 'virtual' assembly, and offer a multitude of advantages within product development. However, a fundamental requirement in the application of any DS methodology is that each structural component is independently characterised, for example, by their free-interface frequency response function (FRF) matrices. It is only with an independent characterisation that structural components can be coupled together in a physically representative manner. Such a characterisation is the focus of this paper.

The free-interface dynamics of a structural component are typically approximated through 'free' suspension. Whilst a suitable approximation is obtained from mid to high frequencies, the low frequency dynamics are often influenced by the presence of resilient supports [4]. Consequently, the acquired FRFs are unsuitable for use within DS.

In the search for an alternative approach, standard DS procedures have been reformulated and used, instead, to decouple structures and return their free-interface dynamics [5, 6, 7]. Whilst this approach, referred to as substructure decoupling (SD), provides an independent characterisation, its implementation requires a priori knowledge of a residual sub-structure, from which the target sub-structure is decoupled. This knowledge is required in the form of the residual's free-interface FRF matrix. Once known, the residual is decoupled from the assembly, leaving behind the free-interface dynamics of the target sub-structure. This standard SD procedure is illustrated diagrammatically in figure 1a.

Whilst SD procedures have gained popularity within the structural dynamic community, they are undeniably inconvenient to implement as they require the dismantling of the assembly beforehand (so as to characterise the residual sub-structure). Furthermore, their application to resiliently coupled assemblies is unclear. This may be illustrated by considering the independent characterisation of a resiliently coupled source sub-structure. Following the standard SD procedure, the residual is taken as either; the receiver sub-structure alone, or as the coupled resilient 
element-receiver portion of the assembly. These are shown diagrammaticality in figures $1 \mathrm{~b}$ and $1 \mathrm{c}$, respectively. In each case there exist clear practical issues. In the former (figure 1b), the residual's free interface dynamics are not readily available from measurement ${ }^{1}$, whilst in the latter (figure 1c), the source is defined such that it includes the resilient supports. Moreover, the argument remains; with standard SD procedures, once dismantled, the residual substructure is no longer under a representative mounting condition. As such, its free-interface dynamics are placed into question.

Clearly, an ideal SD methodology would require only measurements to be made on the assembly in its coupled state, thus avoiding the need for it to be dismantled. The development of such an 'in-situ' approach is the main concern of this paper.
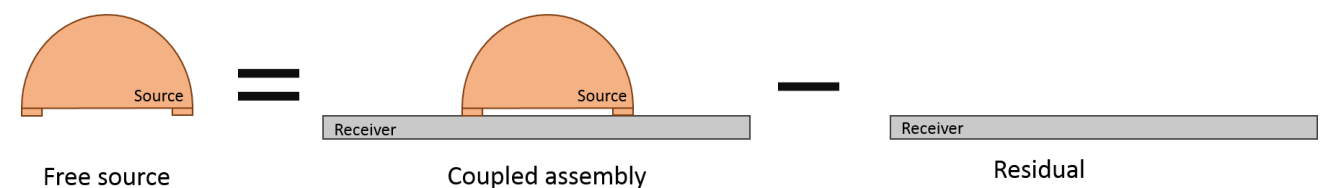

(a) Standard SD applied to a rigidly coupled assembly.

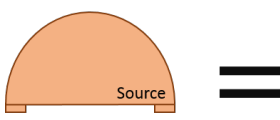

Free source

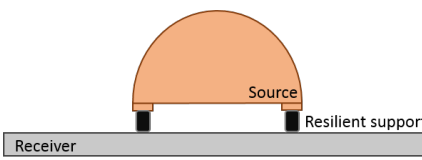

Coupled assembly

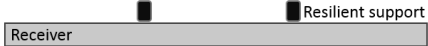

Residual

(b) Standard SD applied to a resiliently coupled assembly. The resdiual is defined as the resilient element-recevier portion of the assembly.
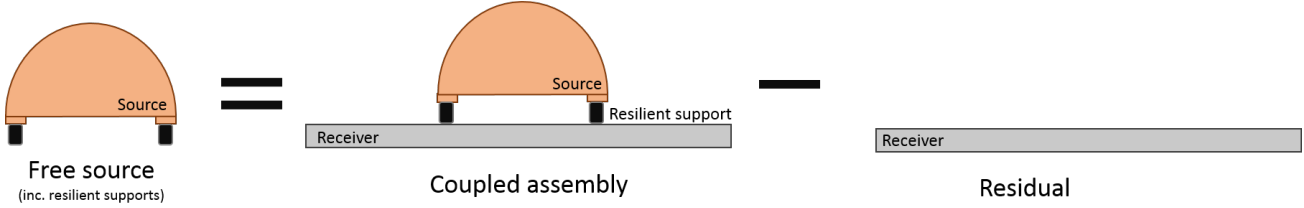

(c) Standard SD applied to a resileint coupled assembly. The residual is defined as the recevier only.

Figure 1: Diagrammatic representations of standard SD procedures applied to rigidly and resiliently coupled assemblies.

Whilst the proposed in-situ SD procedure is restricted to resiliently coupled assemblies ${ }^{2}$, it potentially offers a convenient and independent characterisation of source, receiver and coupling elements simultaneously. Like standard $\mathrm{SD}$, the in-situ decoupling is based on the mathematical removal of a residual sub-structure from the coupled assembly. Unlike standard SD however, the residual here is defined as the resilient supports that couple source and receiver (see figure 2). Through the independent and in-situ characterisation of this residual, the decoupling of source and receiver sub-structures may be achieved without dismantling the assembly.

The in-situ decoupling of resiliently coupled assemblies has previously been investigated by Zhen et al. [8, 9], Pavic and Elliot [10, 11], Wang et al. [12], Keermaekers et al. [13], Liao et al. [14], and Wang et al. [15]. Zhen et al. $[8,9]$ appear to be the first to derive a set of sub-structure decoupling relations. These were later generalised in [12] for more than 2 sub-structures. More recently Wang et al. [15] extended the derivation of Keermaekers et al. [13] such that DoFs remote to the coupling interface may be included in the decoupling procedure. A capability which is recognised in the present work.

\footnotetext{
${ }^{1}$ This would require the measurement of point and transfer mobilities between the free-interfaces of the resilient supports. With the source removed the coupling elements would no longer be under a representative mounting condition. Furthermore, there would likely be little room to perform the required measurements.

${ }^{2}$ It should be noted that the coupling element need not be all that resilient. The SD procedure is more generally restricted to spring-like coupling elements.
} 
In the derivation of their respective formulae, the aforementioned authors assume spring-like coupling elements (i.e. a conservation of force across the element) and present formulas for the free-interface sub-structure FRFs. The SD procedure presented herein is based on this same assumption (conservation of force across the element), and may therefore be considered mathematically equivalent to those discussed above. In contrast to previous works, however, the proposed procedure is formulated from an impedance based sub-structuring perspective, which not only provides a clear and intuitive decoupling, but avoids any lengthy derivations. As for earlier methods, its application provides an independent characterisation of source, receiver and coupling sub-structures, whilst requiring only measurements to be made in-situ on the coupled assembly.

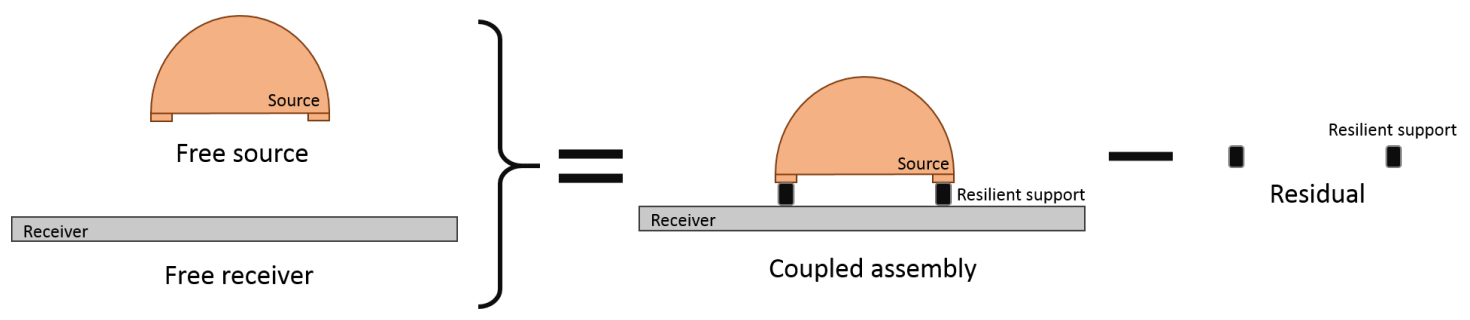

Figure 2: Diagrammatic representation of the in-situ SD procedure applied to a resiliently coupled assembly.

The remainder of this paper will be organised as follows. In section 2 an in-situ approach for the characterisation of resilient coupling elements will be introduced. Making use of this, section 3 will develop the in-situ decoupling procedure. Section 4 will consider a numerical validation of the procedure, before section 5 provides experimental examples. Finally, section 6 draws some concluding remarks.

\section{Independent Characterisation of Coupling Elements In-Situ}

The in-situ decoupling procedure to be outlined later in section 3 relies on the independent characterisation of a residual sub-structure, defined here as the coupling element(s) that lie between source and receiver. This section will focus on the development of an in-situ characterisation method suitable for this purpose.

The preferred quantity for the characterisation of a coupling element is the dynamic transfer stiffness [16]. The dynamic transfer stiffness is an independent property of the coupling element(s) and therefore invariant to changes in the dynamics of source and receiver sub-structures (not including compressional effects such as pre-load). Whilst there exist standardised methods for the determination of dynamic transfer stiffness [17], they require elements to be removed from their intended installation and installed within specialized test rigs. This is not only inconvenient but arguably places the coupling element under a non-representative mounting condition.

With the aim of establishing an in-situ decoupling procedure, an alternative characterisation method is proposed [18]. No assumptions are made with regards to the properties of the source and receiver sub-structures encapsulating the coupling element, other than that they are linear and time invariant. As such, the proposed method facilitates the characterisation of coupling elements whilst in-situ and under representative mounting conditions. This in-situ characterisation will go on to form the basis of the proposed SD procedure, where it is used to independently characterise the residual sub-structure, which is subsequently decoupled from its surrounding assembly.

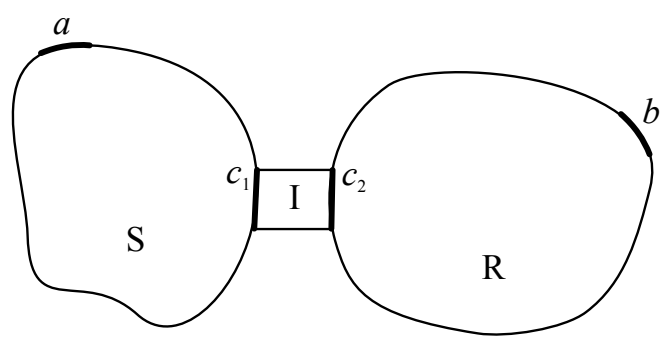

Figure 3: Schematic of the general SIR assembly. 
We begin by considering the interface dynamics of the coupled SIR assembly illustrated in figure 3. Note that I may be composed of multiple coupling elements, and that $c_{1}$ and $c_{2}$ represent the coupling interface DoFs of the source $(\mathbf{S})$ and receiver $(\mathbf{R})$ sub-structures, respectively.

The coupling of structural elements is prescribed through the application of equilibrium and continuity constraints at the appropriate interfaces. This may be expressed mathematically in a number of ways [19]. Here we will consider the classical impedance approach [20], also referred to as the 'primal impedance form', where the coupled impedance is represented as the sum of collocated uncoupled impedances. The dynamics of the coupled assembly illustrated in figure 3 may therefore be given by the expression,

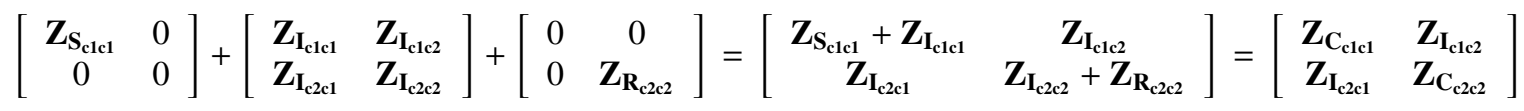

where, $\mathbf{Z}_{\mathbf{S}_{\text {c1c1 }}}$ and $\mathbf{Z}_{\mathbf{R}_{\mathbf{c} 2 \mathbf{2} 2}}$ are the coupling interface impedance matrices of the uncoupled sub-structures $\mathbf{S}$ and $\mathbf{R}$, respectively, and $\mathbf{Z}_{\mathbf{C}_{\mathbf{c l c} 1}}$ and $\mathbf{Z}_{\mathbf{C}_{\mathbf{c} 2 \mathbf{2}}}$ are the coupling interface impedance matrices of the coupled assembly. Similarly, $\mathbf{Z}_{\mathbf{I}_{\mathbf{c l c} 1}}$ and $\mathbf{Z}_{\mathbf{I}_{\mathbf{c} 2 \mathbf{c} 2}}$ are the coupling interface point impedance matrices of the uncoupled element $\mathbf{I}$, and $\mathbf{Z}_{\mathbf{I}_{\mathbf{c} 2 \mathbf{c} 1}}=\mathbf{Z}_{\mathbf{I}_{\mathbf{c l c 2}}}^{\mathbf{T}}$ are their associated transfer impedance matrices.

It is clear from equation 1 that the transfer impedance of $\mathbf{I}$ is invariant to the coupling of $\mathbf{S}$ and $\mathbf{R}$. Further, note that the right hand side of equation 1 represents the coupled assembly's interface impedance matrix, $\mathbf{Z}_{\mathbf{C}}$, and that this may be readily obtained through the inversion of the coupled interface mobility matrix,

$$
\left[\begin{array}{ll}
\mathbf{Z}_{\mathbf{C}_{\mathrm{c} 1 \mathrm{c} 1}} & \mathbf{Z}_{\mathbf{I}_{\mathbf{c} 1 \mathrm{c} 2}} \\
\mathbf{Z}_{\mathbf{I}_{\mathbf{c} 2 \mathrm{c} 1}} & \mathbf{Z}_{\mathbf{C}_{\mathbf{c} 2 \mathrm{c} 2}}
\end{array}\right]=\left[\begin{array}{ll}
\mathbf{Y}_{\mathbf{C}_{\mathrm{clc1}}} & \mathbf{Y}_{\mathbf{C}_{\mathrm{clc} 2}} \\
\mathbf{Y}_{\mathbf{C}_{\mathrm{c} 2 \mathrm{c} 1}} & \mathbf{Y}_{\mathbf{C}_{\mathrm{c} 2 \mathrm{c} 2}}
\end{array}\right]^{-1}
$$

Equation 2 shows that we are able to independently characterise the coupling element $\mathbf{I}$, in terms of its transfer impedance, simply through the inversion of its coupling interface mobility matrix, and extraction of the resultant offdiagonal elements. Once acquired, the transfer impedance may be related to the dynamic transfer stiffness through,

$$
\mathbf{K}_{\mathbf{I}_{\mathbf{c} 1 \mathbf{c} 2}}=i \omega \mathbf{Z}_{\mathbf{I}_{\mathbf{c} 1 \mathbf{c} 2}} \text {. }
$$

This approach has been extensively validated by the authors [18, 21], and shown to provide an independent and broad-band characterisation that is suitable for use within a DS framework [2]. The proposed in-situ characterisation may further benefit those interested in the application of mount stiffness based TPA [22] where, in theory, it may be used to obtain the required mount stiffness of each element whilst installed and under representative mounting conditions, thus avoiding the need to dismantle the assembly. However, this possibility will not be further explored here.

The experimental implementation of equation 2 requires access to the coupling interface to measure the mobility matrix, $\mathbf{Y}_{\mathbf{C}}$. This is often troublesome in practice. The following sub-section aims to provide a solution to this often encountered problem.

\subsection{Extension to Remote Degrees of Freedom}

Whilst laboratory studies may be designed so as to facilitate access at the required interface DoFs, this a luxury not often encountered in practice. The ability to relocate (hard to access) interface excitations to more convenient remote locations would clearly benefit the experimenter.

It was shown by Moorhouse et al. [23] that such a relocation could be achieved through the 'round trip' identity. The round trip identity establishes a relation between the point mobility at a coupling interface, and the transfer mobilities that surround it. It may be used to reconstruct the point mobility at an interface using only remote excitations, that is, avoiding excitation at the interface itself. Whilst the original formulation considered a single coupling interface only, a simple re-derivation is able to provide a dual interface counterpart [21]. For completeness this derivation is presented in Appendix A.

Together, the round trip identity and its dual interface counterpart are able to provide a remote relation for each entry of the coupling interface mobility matrix, $\mathbf{Y}_{\mathbf{C}}$ :

$$
\mathbf{Y}_{\mathbf{C}_{\mathrm{clc1}}}=\mathbf{Y}_{\mathbf{C}_{\mathrm{clb}}} \mathbf{Y}_{\mathbf{C}_{\mathrm{ab}}}^{-1} \mathbf{Y}_{\mathbf{C}_{\mathrm{cla}}}^{\mathbf{T}}
$$




$$
\begin{aligned}
& \mathbf{Y}_{\mathbf{C}_{\text {clc2 }}}=\mathbf{Y}_{\mathbf{C}_{\mathrm{clb}}} \mathbf{Y}_{\mathrm{C}_{\mathrm{ab}}}^{-\mathbf{1}} \mathbf{Y}_{\mathrm{C}_{\mathrm{c} 2 \mathrm{a}}}^{\mathbf{T}} \\
& \mathbf{Y}_{\mathbf{C}_{\mathrm{c} 2 \mathrm{cl}}}=\mathbf{Y}_{\mathbf{C}_{\mathrm{c} 2 \mathrm{a}}} \mathbf{Y}_{\mathrm{Cba}_{\mathrm{b}}}^{-\mathbf{1}} \mathbf{Y}_{\mathbf{C}_{\mathrm{clb}}}^{\mathbf{T}} \\
& \mathbf{Y}_{\mathbf{C}_{\mathrm{c} 2 \mathrm{c} 2}}=\mathbf{Y}_{\mathrm{C}_{\mathrm{c} 2 \mathrm{a}}} \mathbf{Y}_{\mathrm{C}_{\mathrm{ba}}}^{-\mathbf{1}} \mathbf{Y}_{\mathrm{C} \mathrm{cb}}^{\mathbf{T}}
\end{aligned}
$$

where $a$ and $b$ are remote DoFs on the source and receiver, respectively. It should be noted that the above relations do not require any additional assumptions over and above the linearity and time-invariance already assumed.

Substitution of the above into equation 2 yields a remote extension of the in-situ characterisation method.

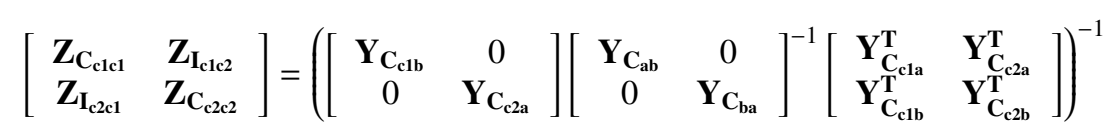

Equation 8 offers an in-situ characterisation of the coupling element $\mathbf{I}$, whilst avoiding all excitations at the coupling interface, and may readily be used in place of equation 2 if convenient (it is valid whether or not access is restricted). Care must be taken that sufficient remote DoFs are used so that the problem is of full rank, that is, the rows and columns of the inverted matrices are sufficiently independent that their inverses may be computed. To this end, additional remote DoFs may be used to provide an over-determined solution.

\section{In-situ Sub-structure Decoupling}

Having established an in-situ method for independently characterising the residual sub-structure, this section will consider the in-situ decoupling of source and receiver.

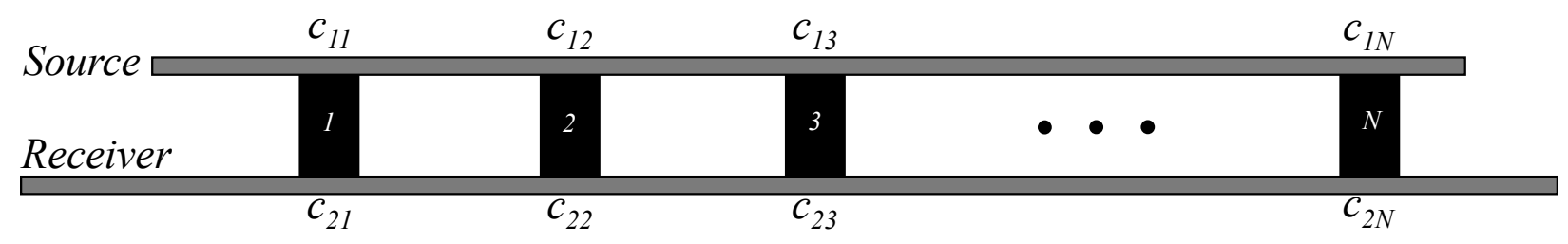

Figure 4: Schematic of the multi-contact coupling interface for an SIR assembly.

Let us again consider the SIR assembly illustrated in figure 3. Shown in figure 4 is a detailed view of the $N$ contact coupling interface. For generality, 6 DoFs are considered at each point. The coupled impedance matrix of the assembly is given by,

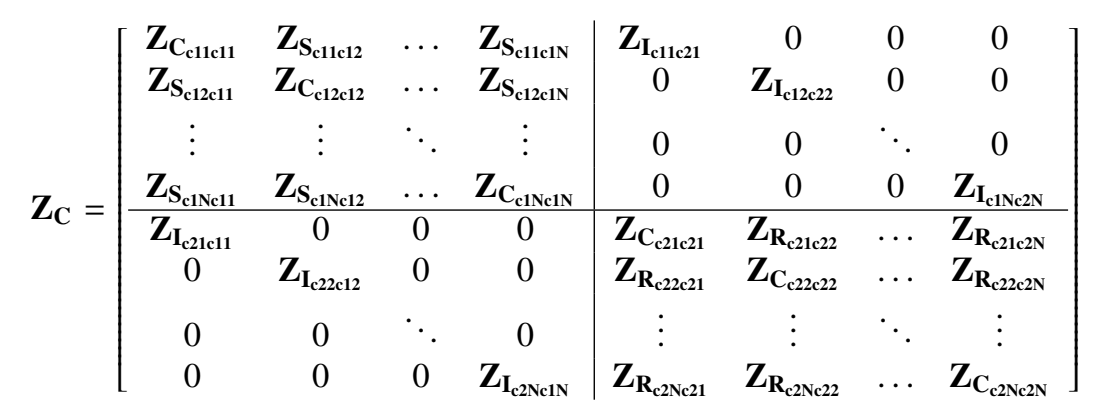

where for example; $\mathbf{Z}_{\mathbf{C}_{\text {c12c12 }}}$ is the coupled point impedance matrix at the second contact on the source sub-structure, $\mathbf{Z}_{\mathbf{S}_{\text {cllciN }}}$ is the coupled transfer impedance matrix between the first and $N$ th contact on the source sub-structure, and $\mathbf{Z}_{\mathbf{I}_{\text {cl1ce21 }}}$ is the coupled transfer impedance matrix across the first coupling element.

From equation 9 it can be seen that the partitioned block diagonal sub-matrices contain both source (S), receiver (R) and assembly $(\mathbf{C})$ impedances. This is a consequence of the point impedance, found along the diagonal, being the sum of source (or receiver) and coupling element(s) impedances, e.g. $\mathbf{Z}_{\mathbf{C}_{\mathrm{clNclN}}}=\mathbf{Z}_{\mathbf{S}_{\mathrm{clNclN}}}+\mathbf{Z}_{\mathbf{I}_{\mathrm{clNclN}}}$. 
Rewriting equation 9 in a further blocked form clearly illustrates that the diagonal elements, $\mathbf{Z}_{\mathbf{C}_{\mathbf{c l c 1}}}$ and $\mathbf{Z}_{\mathbf{C}_{\mathbf{c} 2 \mathrm{c} 2}}$, represent properties of the coupled assembly, whilst the off-diagonal elements, $\mathbf{Z}_{\mathbf{I}_{\mathbf{c} 1 \mathbf{2} 2}}$ and $\mathbf{Z}_{\mathbf{I}_{\mathbf{c} 2 \mathbf{1} 1}}$, are independent properties of the $N$ coupling elements.

$$
\mathbf{Z}_{\mathbf{C}}=\left[\begin{array}{c|c}
\mathbf{Z}_{\mathbf{C}_{\mathrm{clc1}}} & \mathbf{Z}_{\mathbf{I}_{\mathrm{c} 1 \mathrm{c} 2}} \\
\hline \mathbf{Z}_{\mathbf{I}_{\mathrm{c} 2 \mathrm{c} 1}} & \mathbf{Z}_{\mathbf{C}_{\mathrm{c} 2 \mathrm{c} 2}}
\end{array}\right]
$$

With mind to the classical impedance DS formulation (see section 2) [20], equation 10 may be rewritten in the form of a summation using the block diagonal source-receiver impedance matrix,

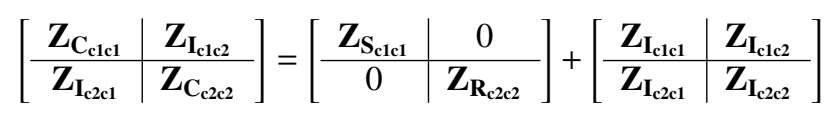

or more condensely as,

$$
\mathbf{Z}_{\mathbf{C}}=\mathbf{Z}_{\mathbf{S R}}+\mathbf{Z}_{\mathbf{I}}
$$

where we note that $\mathbf{Z}_{\mathbf{S R}}$ is the target sub-structure impedance and that $\mathbf{Z}_{\mathbf{I}}$ is the residual.

Decoupling is performed by simply rearranging equation 12 such that the target impedance matrix, $\mathbf{Z}_{\mathbf{S R}}$, is given as the difference between the coupled assembly and residual impedance matrices,

$$
\mathbf{Z}_{\mathbf{S R}}=\mathbf{Z}_{\mathbf{C}}-\mathbf{Z}_{\mathbf{I}}
$$

Unlike standard SD, here the residual is defined as the coupling element(s) I (see figure 2), as opposed to the receiver, or the coupling element(s)-receiver portion of the assembly, as in figures $1 \mathrm{~b}$ and 1c, respectively. By defining the residual as the coupling element(s) we need not dismantle the assembly to perform the residual's independent characterisation. This is instead achieved in-situ, as per section 2, using equation 2 or 8 .

In general, the residual impedance matrix, $\mathbf{Z}_{\mathbf{I}}$, is given by,

$$
\mathbf{Z}_{\mathbf{I}}=\left[\begin{array}{l|l}
\mathbf{Z}_{\mathbf{I}_{\mathbf{c} 1 \mathbf{1} 1}} & \mathbf{Z}_{\mathbf{I}_{\mathbf{c} 1 \mathbf{2} 2}} \\
\hline \mathbf{Z}_{\mathbf{I}_{\mathbf{c} 2 \mathbf{c} 1}} & \mathbf{Z}_{\mathbf{I}_{\mathbf{c} 2 \mathbf{c} 2}}
\end{array}\right]
$$

It was shown in section 2 that the transfer impedances $\mathbf{Z}_{\mathbf{I}_{\mathbf{c l c} 2}}=\mathbf{Z}_{\mathbf{I}_{\mathbf{c} 2 \mathbf{c} 1}}^{\mathbf{T}}$ may be determined through the inversion of a coupled interface mobility matrix. The coupling point impedances, $\mathbf{Z}_{\mathbf{I}_{\mathbf{c l c} 1}}$ and $\mathbf{Z}_{\mathbf{I}_{\mathbf{c} 2 \mathbf{2} 2}}$ are, however, still unknown. Here lies the key assumption of the SD procedure. If we consider the coupling element(s) as spring-like coupling(s), i.e. with negligible distributed mass, the force across the element(s) is conserved and we are able to make the assumption that,

$$
\mathbf{Z}_{\mathbf{I}_{\mathbf{c} 1 \mathbf{c} 1}} \approx-\mathbf{Z}_{\mathbf{I}_{\mathrm{c} 1 \mathbf{c} 2}}=-\mathbf{Z}_{\mathbf{I}_{\mathbf{c} 2 \mathbf{c} 1}^{\mathbf{T}}}^{\mathbf{2}} \approx \mathbf{Z}_{\mathbf{I}_{\mathbf{c} 2 \mathbf{2} 2}},
$$

that is, the point impedance is equal to the negative transfer impedance. The above assumption is only valid for translational $\left(x, y\right.$ and $z$ ) and torsional $\left(\alpha_{z}\right)$ DoFs. Its application to in-line rotational $\left(\alpha_{x}\right.$ and $\left.\alpha_{y}\right)$ DoFs, however, is not clear and warrants further investigation. Nevertheless, assuming that we are able to neglect in-line rotational DoFs, the entire residual impedance matrix may be approximated from the in-situ determined transfer impedance as,

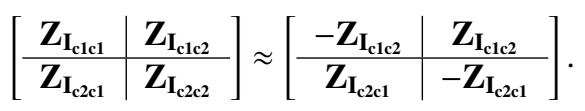

This same assumption, although in a different form, was made by Zhen et al. [8, 9], Pavic and Elliot [10, 11], Keermaekers et al. [13], Liao et al. [14] and Wang et al. [15]. It is, however, worth reiterating that this assumption is only valid whilst the force across the mount is conserved. This assumption is approximately met in the frequency range below the coupling element's first internal resonance.

In practice, it is recognised that at high frequencies the dynamics of resiliently coupled source and receiver substructures often tend to that of a free suspension if the contact area is sufficiently rigid [24]. That is to say, the contribution of impedance from the coupling element(s) will become negligible compared to that of the source and receiver (assuming a sufficiently resilient coupling and inertial source/receiver sub-structure). If this is the case, the 
decoupling procedure will have a minimal effect on the dynamics of the coupled assembly, which are likely to be approximately free.

The in-situ sub-structure decoupling (SD) procedure is summarized by,

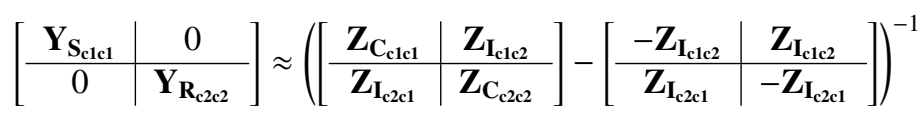

where the entries of the residual impedance matrix are determined via the in-situ characterisation approach (or if access is restricted, via its remote extension), and the coupled impedance matrix is determined simply through the inversion of the coupled mobility matrix (which may also be acquired remotely).

Equation 17 assumes that all DoFs of interest lie at the coupling interfaces, $c_{1}$ and $c_{2}$. Often, however, interest lies in DoFs that are located elsewhere. For example, classical TPA is concerned with the FRFs between coupling interface and interior DoFs, e.g. steering wheel vibration. The in-situ decoupling procedure may readily be extended so as to include these remote DoFs. If we consider the remote source and receiver DoFs, $a$ and $b$, as in figure 3 , equation 17 may be written more generally as,

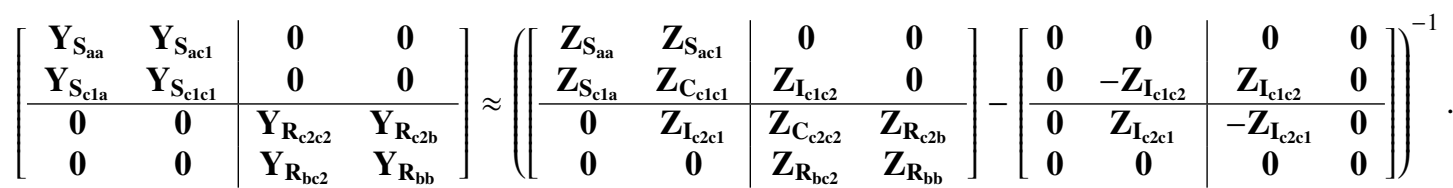

Here, the residual impedance matrix, $\mathbf{Z}_{\mathbf{I}}$, is positioned within an otherwise zero block matrix such that the rows and columns it occupies coincide with those of the coupling DoFs. The purpose of this extension is to provide a free-interface source/receiver FRF matrix that includes the transfer FRFs between coupling and remote DoFs.

It should be noted that the assumption $\mathbf{Z}_{\mathbf{I}_{\mathbf{c} 1 c 1}} \approx-\mathbf{Z}_{\mathbf{I}_{\mathbf{c l c 2} 2}}=-\mathbf{Z}_{\mathbf{I}_{\mathbf{c} 2 \mathbf{2} \mathbf{1}}}^{\mathbf{T}} \approx \mathbf{Z}_{\mathbf{I}_{\mathbf{c} 2 \mathbf{2} 2}}$ is not limited to coupling elements of a resilient nature; it may also be valid for other flexible couplings, provided they too satisfy the conservation of force (for example, an elastic rod below its first internal resonance). As such, equations 17-18 are not strictly limited to resiliently coupled assemblies. Similarly, the in-situ characterisation approach, outlined in section 2, is applicable to non-resilient but flexible elements. However, as the stiffness of the coupling element is increased, practical challenges arise due to the ill-conditioning of the coupling interface mobility matrix. Its application to elements of this type is currently subject to further investigation. If instead the source and receiver are rigidly coupled, the in-situ decoupling procedure is clearly not applicable. For rigidly coupled sub-structures the collocation of $c_{1}$ and $c_{2}$ leads to a singular coupling interface mobility matrix which can not be inverted. For the decoupling of rigidly coupled sub-structures one must rely on standard SD procedures.

\section{Numerical Case Study}

In this section, the in-situ decoupling procedure proposed in section 3 will be validated using a simple numerical mass-spring-dashpot model. The assembly considered is shown diagrammatically in figure 5, with the associated model parameters presented in table 1. The assembly is made up of 3 sub-structures, labelled in figure 5 as source, coupling and receiver. The source and receiver sub-structures are coupled via two spring-dashpot elements and each contain 3 DoFs.

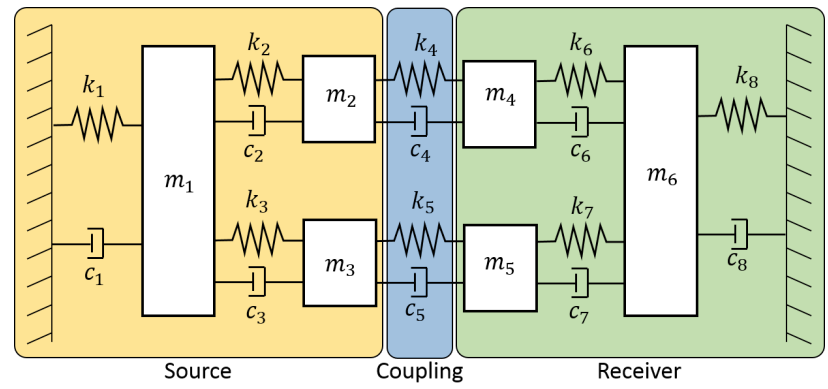

Figure 5: Mass-spring-dashpot model used in the numerical validation of the proposed in-situ decoupling procedure. 
The aim of the numerical study is to identify the free-interface mobility matrices of the source and receiver substructures, using mobilities of the coupled assembly only. Alongside the free-interface mobilities, the impedance of the uncoupled spring-dashpot elements will also be obtained.

\begin{tabular}{cccc}
\hline Mass $(\mathrm{kg})$ & Damping $\left(\mathrm{N} / \mathrm{ms}^{-1}\right)$ & Stiffness $(\mathrm{N} / \mathrm{m})$ & Impedance $\left(\mathrm{N} / \mathrm{ms}^{-1}\right)$ \\
\hline$m_{1}=0.1$ & $c_{1}=10$ & $k_{1}=1 \times 10^{5}$ & \\
$m_{2}=0.2$ & $c_{2}=20$ & $k_{2}=2 \times 10^{5}$ & \\
$m_{3}=0.4$ & $c_{3}=30$ & $k_{3}=3 \times 10^{5}$ & \\
$m_{4}=0.2$ & $c_{4}=50$ & $k_{4}=7 \times 10^{5}$ & $Z_{24}=50-i \frac{7 \times 10^{5}}{\omega}$ \\
$m_{5}=0.3$ & $c_{5}=60$ & $k_{5}=8 \times 10^{5}$ & $Z_{35}=60-i \frac{8 \times 10^{5}}{\omega}$ \\
$m_{6}=0.5$ & $c_{6}=10$ & $k_{6}=1 \times 10^{5}$ & \\
& $c_{7}=20$ & $k_{7}=2 \times 10^{5}$ & \\
& $c_{8}=30$ & $k_{8}=3 \times 10^{5}$ & \\
\hline
\end{tabular}

Table 1: Model parameters used in the numerical simulation. The impedance values given correspond to those of the coupling element only (spring-dashpot), i.e do not include inertial effects of neighbouring masses.

For the assembly considered, the coupled mobility is given by,

$$
[\mathbf{Y}]=\left(i \omega[\mathbf{M}]+[\mathbf{C}]+\frac{1}{i \omega}[\mathbf{K}]\right)^{-1}
$$

where the mass, damping and stiffness matrices are each given, respectively, by

$$
\begin{gathered}
{[\mathbf{M}]=\operatorname{diag}\left(\begin{array}{cccccc}
m_{1}, & m_{2}, & m_{3}, & m_{4}, & m_{5}, & m_{6}
\end{array}\right),} \\
{[\mathbf{C}]=\left[\begin{array}{cccccc}
c_{1}+c_{2}+c_{3} & -c_{2} & -c_{3} & 0 & 0 & 0 \\
-c_{2} & c_{2}+c_{4} & 0 & -c_{4} & 0 & 0 \\
-c_{3} & 0 & c_{3}+c_{5} & 0 & -c_{5} & 0 \\
0 & -c_{4} & 0 & c_{4}+c_{6} & 0 & -c_{6} \\
0 & 0 & -c_{5} & & c_{5}+c_{7} & -c_{7} \\
0 & 0 & 0 & -c_{6} & -c_{7} & c_{6}+c_{7}+c_{8}
\end{array}\right],}
\end{gathered}
$$

and

$$
[\mathbf{K}]=\left[\begin{array}{cccccc}
k_{1}+k_{2}+k_{3} & -k_{2} & -k_{3} & 0 & 0 & 0 \\
-k_{2} & k_{2}+k_{4} & 0 & -k_{4} & 0 & 0 \\
-k_{3} & 0 & k_{3}+k_{5} & 0 & -k_{5} & 0 \\
0 & -k_{4} & 0 & k_{4}+k_{6} & 0 & -k_{6} \\
0 & 0 & -k_{5} & & k_{5}+k_{7} & -k_{7} \\
0 & 0 & 0 & -k_{6} & -k_{7} & k_{6}+k_{7}+k_{8}
\end{array}\right]
$$

The in-stu decoupling procedure requires first the construction of the residual impedance matrix, $\mathbf{Z}_{\mathbf{I}}$. This is done using the in-situ characterisation approach presented in section 2. Having acquired the transfer impedance of the coupling elements using the in-situ approach, the residual impedance matrix is constructed as per equation 16 . For the spring-dashpot coupling elements considered, the residual impedance matrix is obtained exactly. The approximate form of equation 16 occurs when the coupling elements are not pure spring-like, such as when there is distributed mass in the elements. With massless couplings considered, this assumption is avoided and an exact impedance is found.

The source and receiver sub-structures each contain a single remote DoF, i.e. those corresponding to mass 1 and mass 6 , respectively. The aim is to determine the free-interface mobility matrices that include these remote DoFs. As such, the residual impedance matrix must be modified as per equation 18 , where it is positioned within an otherwise null matrix. Implementation of equation 18 consequently yields the free-interface mobility matrices of the source and receiver sub-structures. Some example entries of these mobility matrices are shown in figure 6. Also shown are 
the mobilities acquired from the physically uncoupled sub-structures, modelled numerically. It can be seen that, for theoretical mobilities and massless coupling elements, the in-situ decoupled mobilities are in exact agreement with those of the physically uncoupled sub-structures.

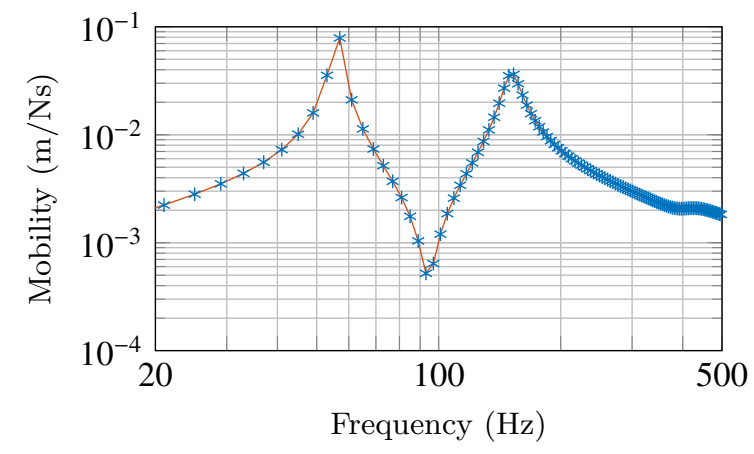

(a) Source point mobility $-Y_{S_{22}}$

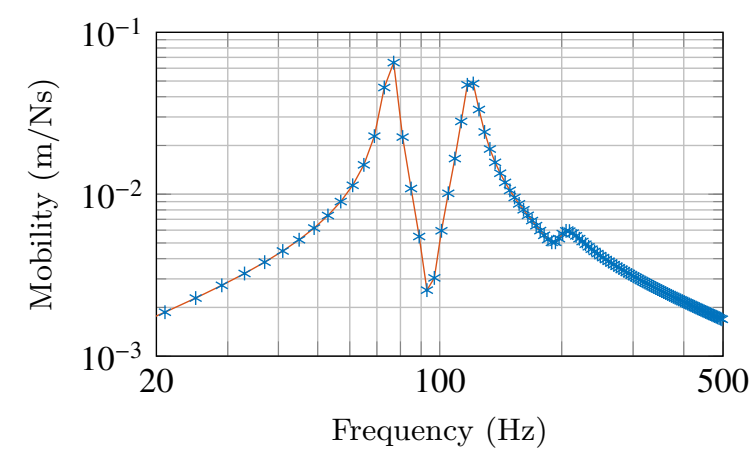

(c) Recevier point mobility - $Y_{R_{44}}$

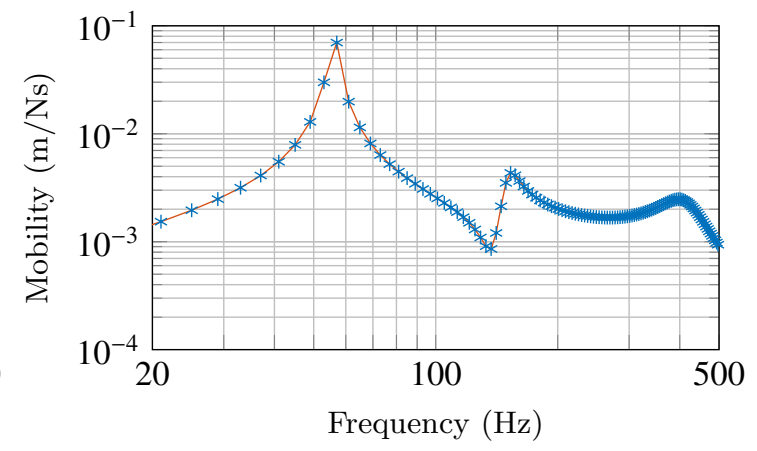

(b) Source transfer mobility $-Y_{S_{21}}$

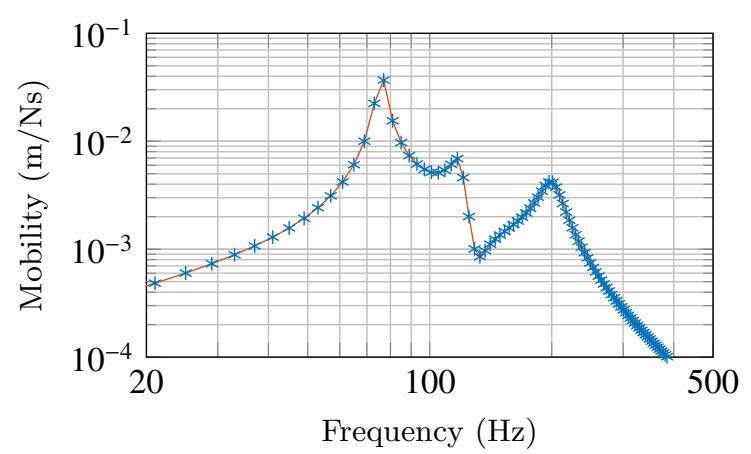

(d) Recevier transer mobility - $Y_{R_{46}}$

Figure 6: Free-interface source and receiver mobilities acquired using the in-situ decoupling procedure (orange) and physical uncoupling (blue stars).

It is worth noting that the proposed in-situ decoupling procedure not only successfully determined the freeinterface mobility matrices of the source and receiver sub-structures, including the remote DoFs associated with $m_{1}$ and $m_{6}$, but acquired them simultaneously. This is an advantage over the alternative approaches of $[10,11,13,14]$ where only the free-interface coupling DoFs are acquired, even then for only a single sub-structure at a time. Furthermore, the proposed approach allowed for the extraction of the coupling element(s) transfer impedance. The above results validate the proposed in-situ decoupling procedure for simple spring-dashpot like couplings and theoretically obtained mobilities. Its application in a more realistic and practical scenario using measured data is considered in the following section.

\section{Experimental Case Studies}

With the in-situ decoupling procedure having been validated numerically in section 4 , this section will consider its experimental implementation. Three experimental studies have been conducted. In each, the free-interface FRFs of source and receiver sub-structures are determined via the in-situ decoupling procedure. Shown in figure 7 are diagrammatic representations of the experimental studies presented. Two single contact assemblies; a mass-isolatorplate (MIP) and a beam-isolator-plate (BIP), and a single multi-contact beam-isolator-isolator-plate (BIIP) assembly were considered. Rubber type isolators were used in all studies. 
Whilst the proposed in-situ decoupling procedure facilitates decoupling in multiple translationary coordinateDoFs, the presented studies consider only the decoupling of sub-structures in translational $z$ DoFs. Application of the procedure to additional coordinate-DoFs is subject to further investigation.

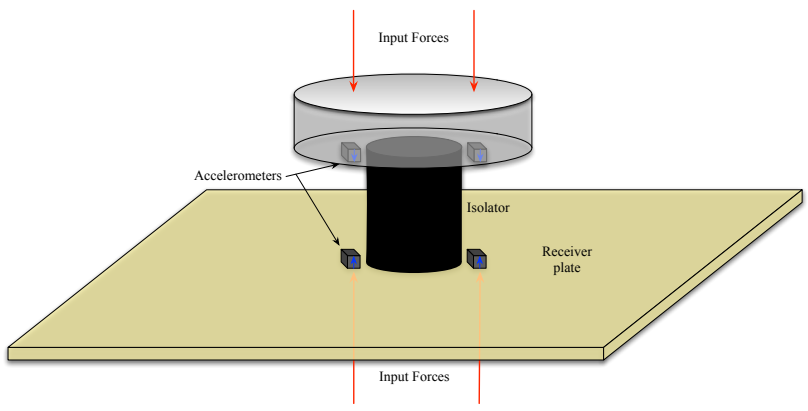

(a) Mass-Isolator-Plate (MIP)

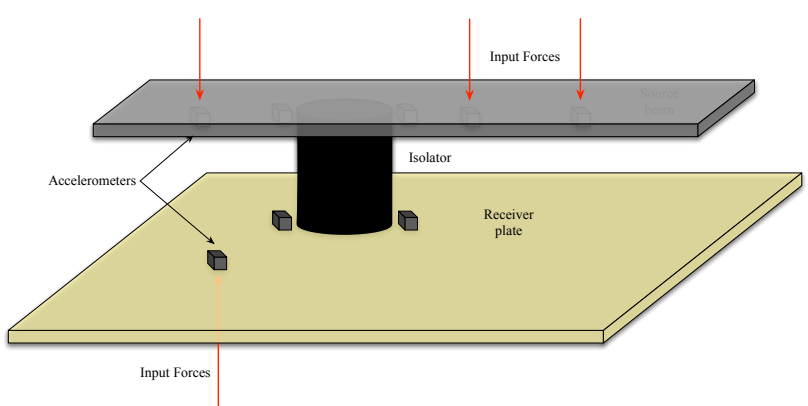

(c) Beam-Isolator-Plate (remote extension)

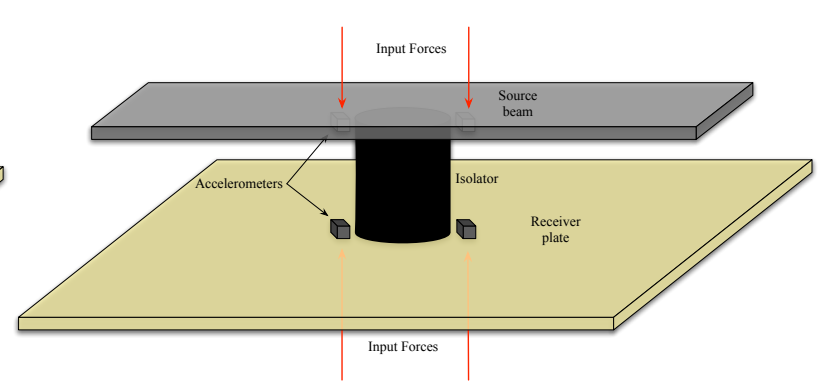

(b) Beam-Isolator-Plate (BIP)

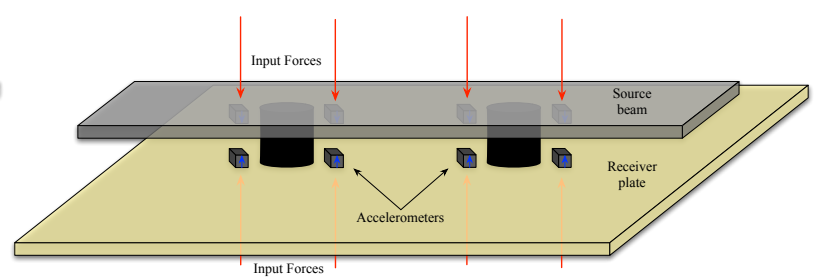

(d) Beam-Isolator-Isolator-Plate (BIIP)

Figure 7: Diagrammatic representation of the experimental assemblies considered.

\subsection{Mass-Isolator-Plate}

As per the procedure outlined in section 3, the decoupling of the MIP assembly (figure 7a) first requires the measurement of its coupling interface mobility matrix. This was achieved through the averaging of a spaced mobility matrix, obtained from the measurement illustrated in figure 7a. Once acquired the coupling interface mobility matrix was inverted, yielding the coupled assembly's impedance matrix, $\mathbf{Z}_{\mathbf{C}}$. From this, the transfer impedances were extracted and used to construct the approximate residual impedance matrix $\mathbf{Z}_{\mathbf{I}}$, according to equation 16 . The block diagonal source-receiver free-interface mobility matrix, $\mathbf{Y}_{\mathbf{S R}}$, was subsequently obtained via equation 17.

The dynamic transfer stiffnesses of the coupling element, obtained via the in-situ characterisation approach presented in section 2, are shown in figure 8a. The acquired stiffnesses clearly exhibit the expected trends, that is, a near linear frequency response with a well damped internal resonance. A broad-band characterisation is observed with convincing results obtained over the range of approximately $30-3000 \mathrm{~Hz}$. Above $3 \mathrm{kHz}$ an increasing amount of noise is observed. This is a result of signal attenuation through the coupling element, which causes some measured mobilities to fall below the equipment noise floor. The use of more sensitive accelerometers would likely mitigate the severity of this effect. A more thorough validation of the in-situ characterisation approach itself is presented in $[18,21]$. For now, the results presented in figure 8a serve to demonstrate the in-situ characterisation of the residual. Note that the stiffnesses acquired for the case studies presented in section 5.2 and 5.3 are comparable in quality to that presented here, but will not be shown for brevity [21].

Shown in figure $8 \mathrm{~b}$ are the coupled (orange) and in-situ decoupled (green) accelerances of the source mass. Accelerances are shown for this particular source to better illustrate its mass like nature. It can clearly be seen that much of the low frequency resonant behaviour observed in the coupled response (green) has been removed. The remaining dynamics appear more in keeping with that expected from a mass like structure, i.e. a constant accelerance. Whilst 


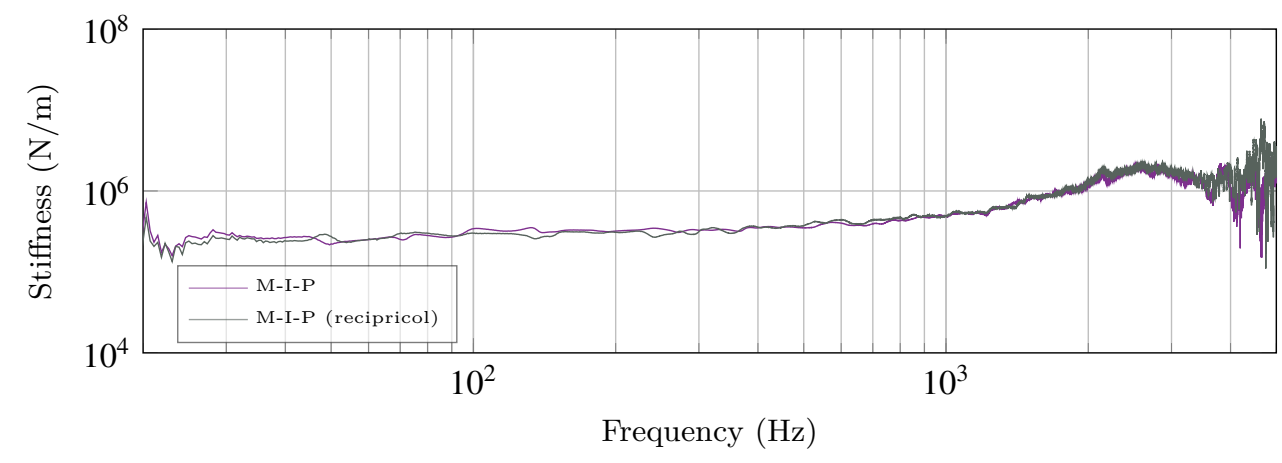

(a) Transfer impedance $\mathbf{Z}_{\mathrm{cl} \mathbf{2}}$ and $\mathbf{Z}_{\mathrm{c} \mathbf{c} 1}$ of the coupling element aquired from the Mass-Isolator-Plate assembly. (Shown up to $5 \mathrm{kHz}$ to cover internal resonance)

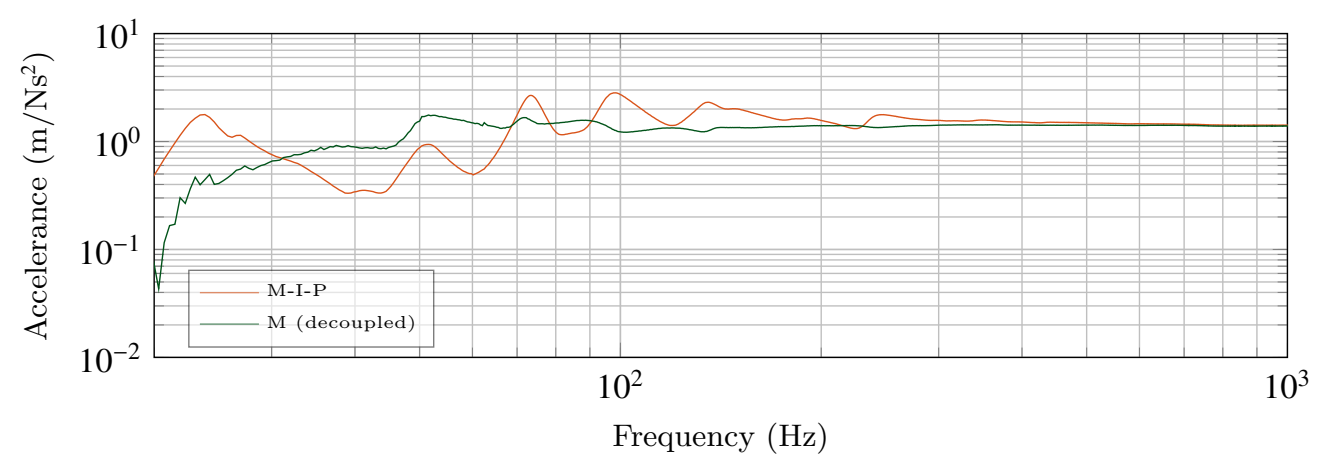

(b) Accelerance $\mathbf{A}_{\text {c1c1 }}$ of the coupled (orange) and in-situ decoupled (green) source mass.

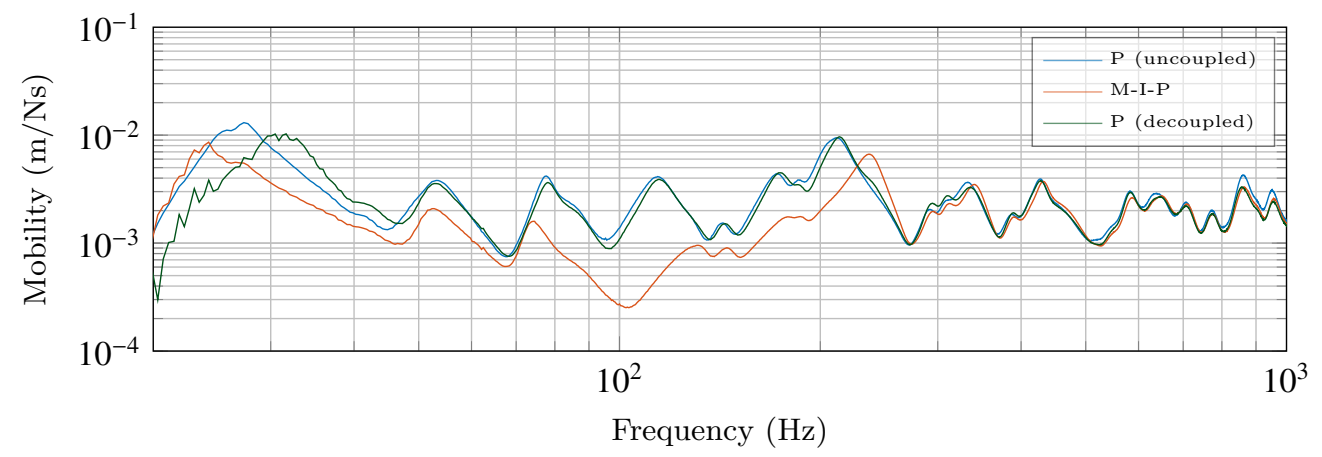

(c) Mobility $\mathbf{Y}_{\mathbf{c 2} 22}$ of the coupled (orange), physically uncoupled (blue), and in-situ decoupled (green) recevier plate.

Figure 8: In-situ decoupling results for the Mass-Isolator-Plate assembly shown in figure 7a 
we do not have a physically uncoupled accelerance for comparison ${ }^{3}$, it is clear that the resonant behaviour observed in the coupled accelerance does not belong to the source. A reduction in this resonant behaviour, and extension of the constant accelerance, suggests that the SD procedure has been at least partially successful.

Shown in figure 8c are the coupled (orange), in-situ decoupled (green), and physically uncoupled (blue) mobilities of the receiver plate. The success of the SD procedure is clearly illustrated here. Below approximately $250 \mathrm{~Hz}$ the coupled and physically uncoupled mobilities are seen to differ considerably. ${ }^{4}$ The in-situ decoupled prediction, however, is in excellent agreement with that of the physically uncoupled mobility. This agreement extends above approximately $40 \mathrm{~Hz}$. Above $250 \mathrm{~Hz}$ the coupled and in-situ decoupled mobilities converge with the physically uncoupled mobility. This is a result of the negligible impedance of the coupling element. Consequently, at high frequencies the free-interface dynamics are suitably approximated by the coupled dynamics. This convergence further suggests that whilst the massless coupling assumption breaks down in the region of the internal mount resonance (approx $3 \mathrm{kHz}$, as shown in figure 8a), the coupling element contributes a negligible impedance and so the procedure remains valid towards this region.

\subsection{Beam-Isolator-Plate}

Having successfully applied the in-situ decoupling procedure on a partly resonant Mass-Isolator-Plate assembly, a more challenging Beam-Isolator-Plate (BIP) assembly is considered. The resonant nature of this assembly may be considered a more realistic representation of what could be encountered in a practical scenario.

Following the same procedure as described in section 5.1, the in-situ decoupling of the BIP assembly requires first the measurement of its coupling interface mobility matrix. In this study, the mobility matrix was determined both directly, and remotely via equations 4-7. This remote determination avoided the need to excite either coupling interface.

The direct measurement of the coupling interface mobility matrix was preformed by averaging a spaced mobility matrix, obtained from the measurement illustrated in figure $7 \mathrm{~b}$. Remote measurements were preformed as illustrated in figure 7c, and used to calculate the mobility matrix as per equations 4-7. A pair of accelerometers were positioned about the coupling interface with an additional 4 placed at remote DoFs ( 3 on the source $(a)$ and 1 on the receiver $(b)) .^{5}$ Forces were applied only at these remote DoFs, that is, no forces were applied at the coupling interface. The resulting coupling interface mobility matrices (direct and remote) were inverted and the off-diagonal transfer impedances used to construct the approximate residual impedance matrix, $\mathbf{Z}_{\mathbf{I}}$. Decoupling was then preformed as per equation 17 .

Shown in figure 9a are the coupled (orange) and in-situ decoupled (green) mobilities of the source beam. Also shown is the in-situ decoupled source mobility acquired using the remote extension (dashed green). Although the freeinterface source mobility is unavailable for comparison, a number of promising trends can be observed. It can be seen that below approximately $300 \mathrm{~Hz}$, the resonant behaviour observed in the coupled mobility is removed almost entirely. The resulting mobility exhibits a near linear low frequency response, as expected from the rigid body behaviour of a free-free beam. Furthermore, the decoupling procedure yields a mobility considerably less damped than that of the coupled assembly, as illustrated by the $750 \mathrm{~Hz}$ resonance. Again, this is expected as the coupling element would introduce considerable damping to the assembly.

The remote decoupling can be seen to predict an anti-resonance slightly lower in frequency than that of the coupled and directly decoupled mobilities. This is likely due to an error in the remote measurement of the coupling interface mobility matrix. An additional error can be observed around $900 \mathrm{~Hz}$, although in this region the dynamics of the coupled assembly are approximately free, regardless, so this is not considered an issue. It is believed that further over-determination, or relocation of the remote DoFs would help reduce the severity of this error.

Shown in figure $9 \mathrm{~b}$ are the coupled (orange), in-situ decoupled (green) and physically uncoupled (blue) mobilities of the receiver plate. Also shown is the in-situ decoupled receiver mobility acquired using the remote extension (dashed green). Again, good agreement is obtained between the in-situ decoupled and physically uncoupled receiver mobilities. Although the agreement is not that of the MIP assembly, it clearly illustrates that the in-situ decoupled

\footnotetext{
${ }^{3}$ Any attempt to measure the true free-interface dynamics of a source sub-structure will require some form of resilient suspension. As such the true free-interface dynamics are not available through direct measurement.

${ }^{4}$ This highlights the sort of deviation one might expect by using the mobility of a resiliently coupled structure in place of its free-interface mobility, an approximation that is often made.

${ }^{5}$ The use of three source DoFs allowed for the over determination of the round trip identities.
} 
mobility provides a superior estimation of the true free-interface mobility. Figure $9 \mathrm{~b}$ further illustrates that, using the remote extension, it is possible to successfully decouple the assembly whilst avoiding the need for any interface excitations.

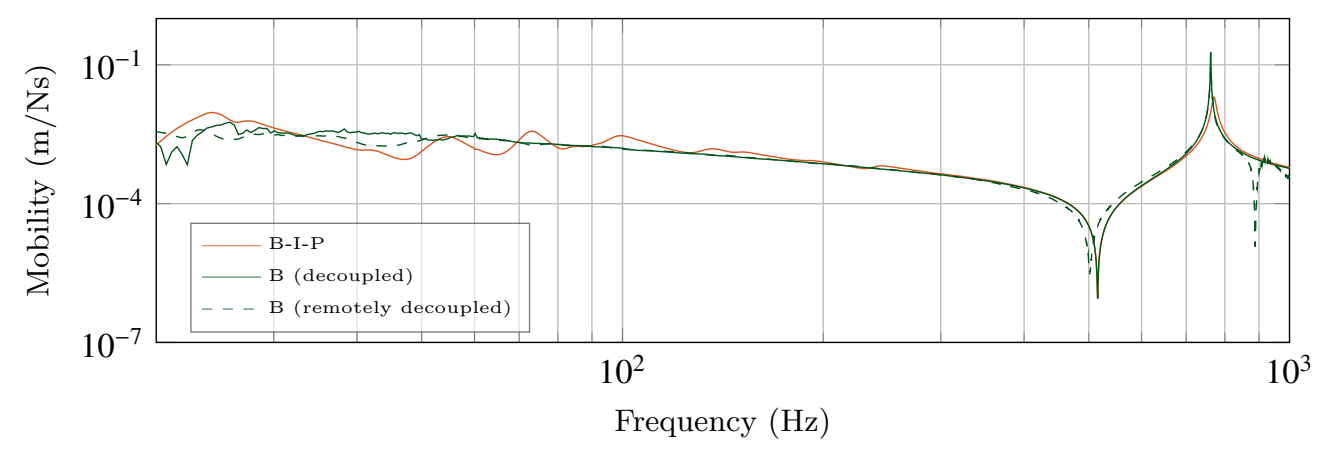

(a) Mobility $\mathbf{Y}_{\mathbf{c l c 1}}$ of the coupled (orange) and in-situ decoupled (green) source beam.

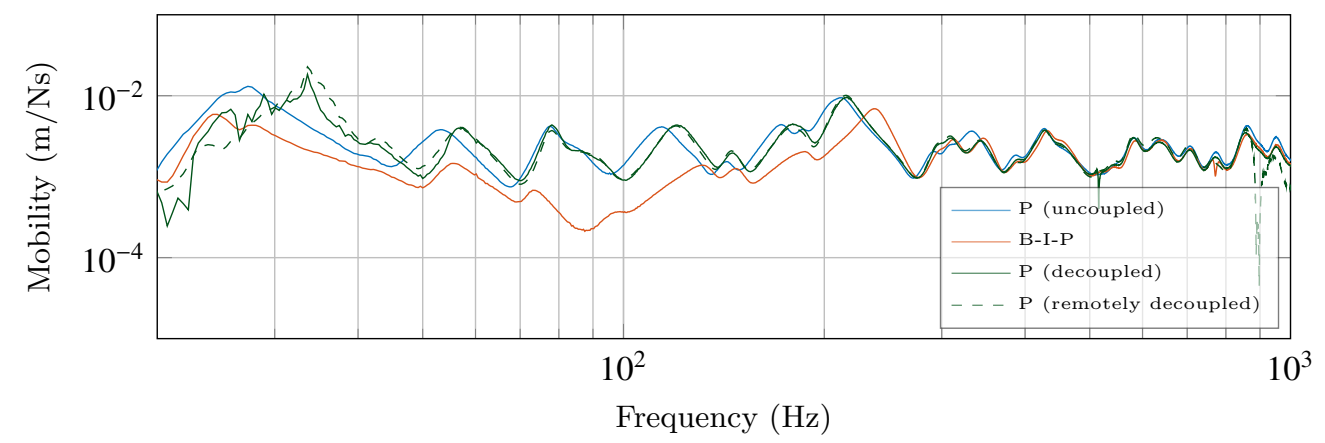

(b) Mobility $\mathbf{Y}_{\mathbf{c} 2 \mathbf{c} 2}$ of the coupled (orange), physically uncoupled (blue), and in-situ decoupled (green) receiver plate.

Figure 9: Coupled, physically uncoupled and in-situ decoupled point mobilities of the beam-isolator-plate assembly shown in figure 7b. In-situ decoupled mobilities are presented for both direct (solid) and remote (dashed) approaches.

Together, figures 8 and 9 demonstrate that it is possible to acquire good estimates of the free-interface FRFs of resiliently coupled sub-structures based on measurements made in-situ on the coupled assembly.

\subsection{Beam-Isolator-Isolator-Plate}

The case studies presented thus far have considered the decoupling of sub-structures that are coupled via only a single element. In practice, real structures are likely to be coupled via multiple elements. This study concerns a Beam-Isolator-Isolator-Plate (BIIP) assembly (figure 7d), and demonstrates the in-situ decoupling of a multi-contact coupled structure.

With the introduction of a second coupling DoF the decoupling procedure requires both coupling elements to be included in the coupling interface mobility matrix. This matrix was measured directly using spaced accelerometer/force pairs at each contact interface, as shown in figure $7 \mathrm{~d}$. Once measured, the coupling interface mobility matrix was inverted and the off diagonal elements used to construct the residual impedance matrix, $\mathbf{Z}_{\mathbf{I}}$. The decoupling procedure was then implemented as per equation 17.

With the assembly now having two coupling DoFs, the mobility matrix, $\mathbf{Y}_{\mathbf{C}}$, has the dimensions $4 \times 4$. Consequently, the block transfer impedance matrix, $\mathbf{Z}_{\mathbf{I}_{\mathbf{c l c} 2}}$, has the dimensions $2 \times 2$, with the transfer impedance of each coupling element along its diagonal. These diagonal elements are extracted and used to construct the approximate the residual impedance matrix. 


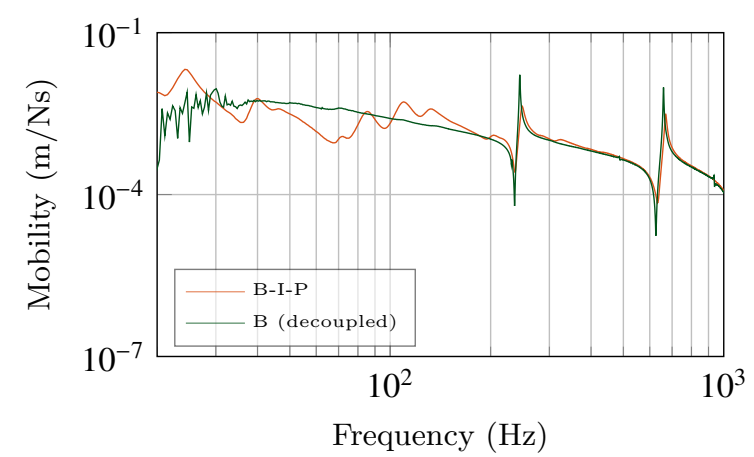

(a) $\mathbf{Y}_{\text {c11c11 }}$

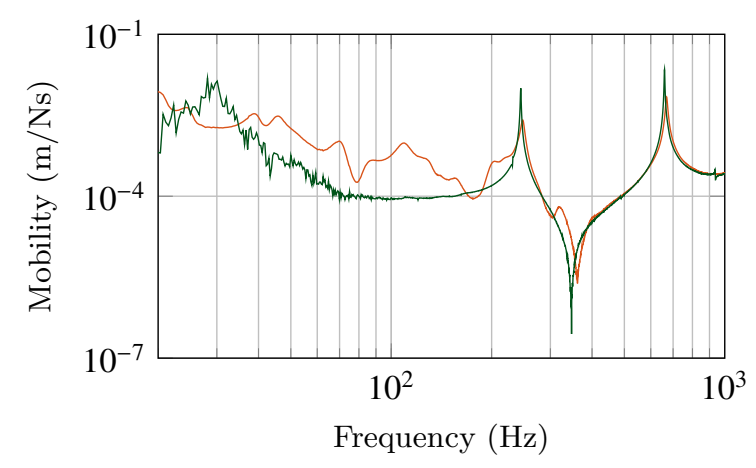

(c) $\mathbf{Y}_{\mathrm{c12c11}}$

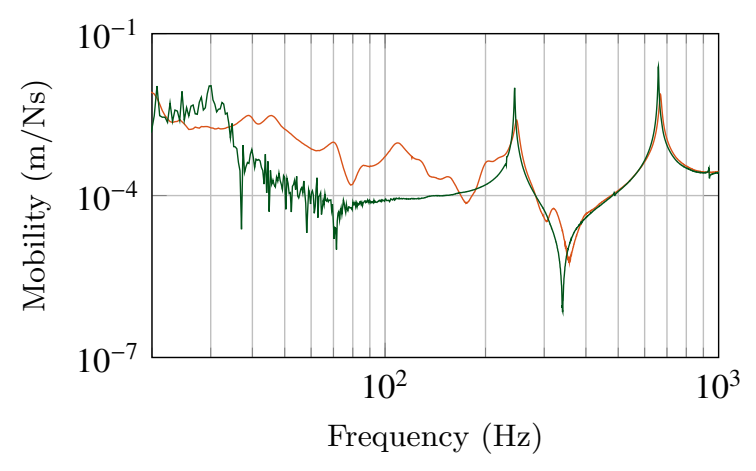

(b) $\mathbf{Y}_{\text {c11c12 }}$

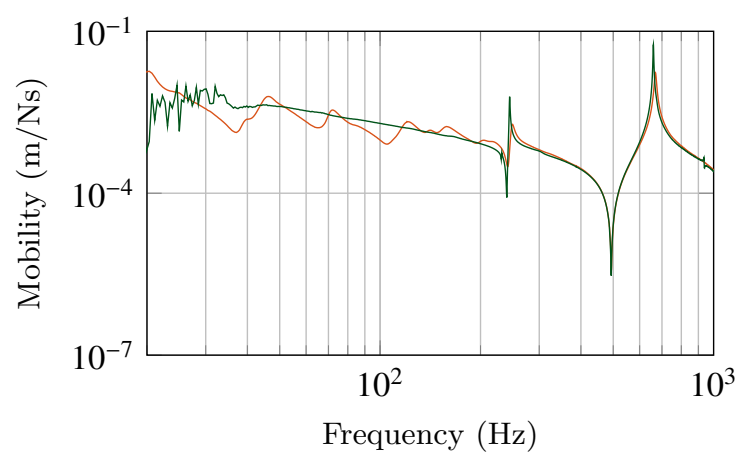

(d) $\mathbf{Y}_{\mathbf{c 1 2} \mathbf{c} 12}$

Figure 10: Coupled (orange) and in-situ decoupled (green) source mobilities of the Beam-Isolator-Isolator-Plate assembly shown in figure 7d.

Shown in figure 10 are the coupled (orange) and in-situ decoupled (green) mobility matrices of the source beam. A number of promising improvements are observed through the in-situ decoupling. The low frequency resonant behaviour, occurring below approximately $400 \mathrm{~Hz}$, is almost entirely removed in both point and transfer mobilities, leaving behind source mobilities with the expected low frequency trends. Furthermore, as in the previous study, the decoupling procedure can be seen to significantly reduce the effect of damping in the first few resonances (around $250 \mathrm{~Hz}$ and $650 \mathrm{~Hz}$ ). Lastly, as one would expect, the in-situ decoupled mobility converges with that of the coupled mobility with increasing frequency, suggesting that the impedance of the coupling elements becomes less significant at higher frequencies.

Shown in figure 11 are the coupled (orange), in-situ decoupled (green) and physically uncoupled (blue) mobility matrices of the receiver plate. Although the in-situ decoupled and physically uncoupled mobilities are not in as close agreement as those of the single contact cases, figure 11 clearly shows that the decoupled prediction offers an improved estimation of the free-interface receiver mobility matrix, compared to that of the resiliently coupled case. 


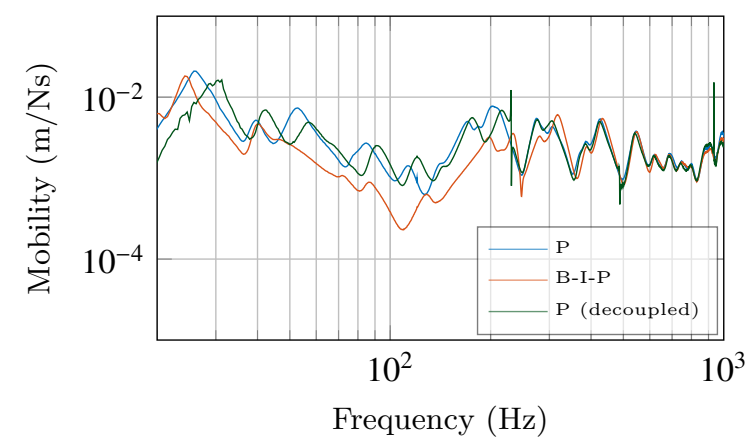

(a) $Y_{\text {c21c21 }}$

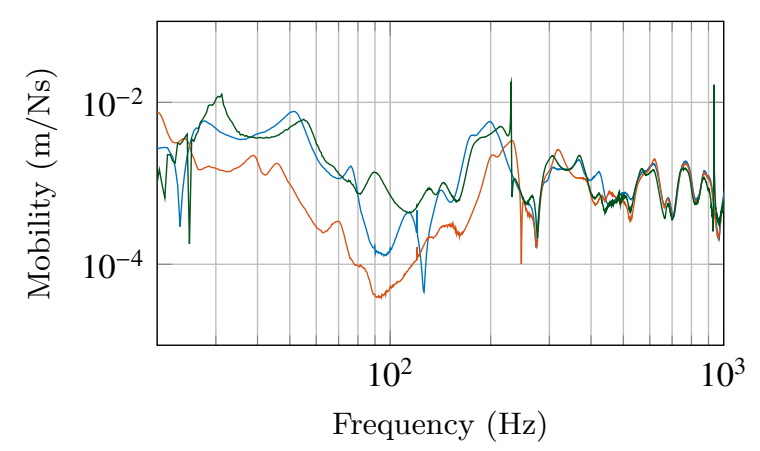

(c) $\mathbf{Y}_{\mathbf{c 2 2} 2 \text { 21 }}$

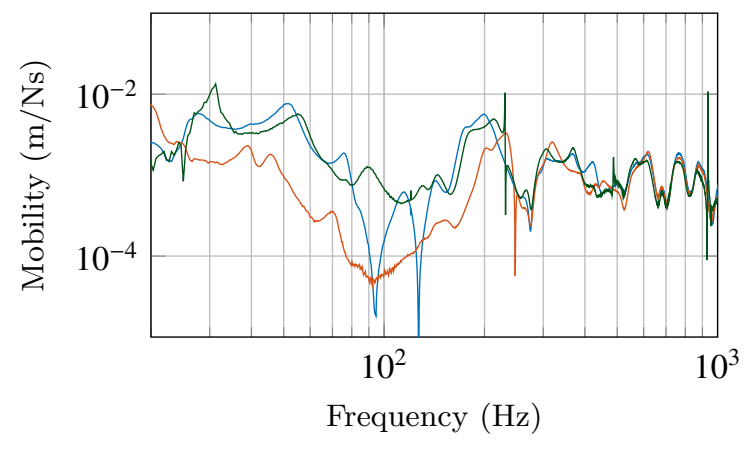

(b) $\mathbf{Y}_{\text {c21c22 }}$

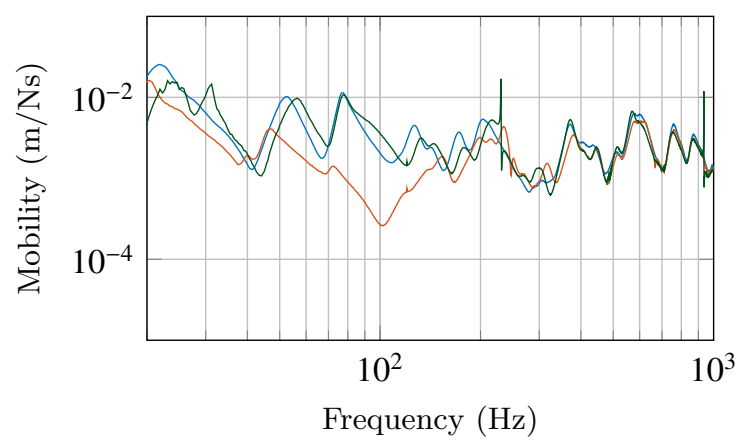

(d) $\mathbf{Y}_{\mathbf{c 2 2} 222}$

Figure 11: Coupled (orange), physically uncoupled (blue), and in-situ decoupled (green) receiver mobilities of the Beam-Isolator-Isolator-Plate assembly shown in figure $7 \mathrm{~d}$.

Whilst the in-situ decoupled prediction can be seen to follow the general trend of the uncoupled mobility, there clearly exist a number of errors. The most noticeable are located about the $100 \mathrm{~Hz}$ transfer mobility anti-resonances in $\mathbf{Y}_{\mathbf{c} 21 \mathbf{c 2} 2}$ and $\mathbf{Y}_{\mathbf{c 2 2 c 2 1}}$. As shown in figure $11 \mathrm{~b}$ and 11c the in-situ decoupled mobility fails to accurately predict these anti-resonances. That said, this error is not necessarily the fault of in-situ decoupling procedure and may result from; experimental error in the measurement of mobilities, neglected DoFs (i.e. rotational and/or in-plane), or perhaps changes in the properties of the receiver due to coupling (e.g. a stiffening due to multiple contacts). Also introduced are a number of sharp resonant artefacts, most notably at approximately $200 \mathrm{~Hz}$. This is a result of artefacts in the direct determination of the coupling element transfer impedance. It has been shown that this error can be avoided by using the remote extension [18].

In-spite of the errors encountered, figures 10 and 11 illustrate the application of the proposed SD procedure to a multi-contact coupled structure and, furthermore, show that it can be used to obtain an improved estimate of the sub-structures' free-interface dynamics.

\section{Concluding Remarks}

This paper has introduced an in-situ dynamic sub-structure decoupling (SD) procedure suitable for resiliently coupled assemblies. Based on a novel in-situ characterisation method for coupling elements, the decoupling procedure allows for the source, coupling element and receiver sub-structures, of an arbitrary assembly, to be mathematically decoupled. Unlike standard SD procedures, the proposed method does not require the assembly to physically dismantled, as it relies upon in-situ measurements only.

Further to the in-situ characterisation and decoupling procedure, the round trip identity and its dual interface counterpart were introduced. Together they allow for the coupling interface mobility matrix of an assembly to be acquired 
without having to apply any excitations at the coupling interface. This facilitates an extension of the decoupling procedure by relocating interface excitations to remote DoFs on the source and receiver sub-structures.

The decoupling procedure was validated numerically using a mass-spring-dashpot model, where it was shown that, for simple couplings (spring-dashpots), the entire free-interface mobility matrices of the source and receiver could be found using coupled mobilities only. The experimental application of the proposed procedure was subsequently investigated, where a number of single and multi-contact assemblies were considered. Results clearly demonstrate that the SD procedure is able to decouple resiliently coupled assemblies using only in-situ measurements. The acquired free-interface mobilities where in good agreement with those expected from theory, or measured directly. It was further shown that the remote extension is able to provide a reasonable prediction of the free-interface mobilities, whilst avoiding any interface excitations.

The independent characterisations (of source, receiver and coupling element) provided by the proposed SD are compatible with standard DS procedures and may therefore be used in the construction of virtual assemblies and acoustic prototypes $[25,2]$.

Further to the independent characterisation of structural components (for use within DS and VAPs), the proposed SD procedure lends itself to use within classical and mount stiffness based transfer path analysis (TPA) [22]. The former requires a vehicle to be dismantled in order to measure the uncoupled FRF matrix required for an input force estimation. The SD procedure would in theory allow for this FRF matrix to be acquired from in-situ measurements, provided that the source regions were resiliently coupled. In the latter the stiffness of coupling mounts are used to estimate input forces. The in-situ residual characterisation developed in this work would in theory allow for the mount stiffness to be acquired in-situ, thus avoiding the need to remove them from the vehicle.

\section{Appendix A. Dual Interface Round Trip}

Here we will derive a dual interface round trip relation following a similar approach to that of Moorhouse et al. [23]. We begin by considering the general SIR assembly illustrated in figure 3. The resultant velocities on the coupled assembly at DoFs $a, c_{1}$, and $c_{2}$, due to an applied force at $b$ are given by,

$$
\begin{aligned}
\mathbf{v}_{\mathbf{C}_{\mathbf{a}}} & =\mathbf{Y}_{\mathbf{C}_{\mathrm{ab}} \mathbf{f}_{\mathbf{C}_{\mathrm{b}}}} \\
\mathbf{v}_{\mathbf{C}_{\mathrm{c} 1}} & =\mathbf{Y}_{\mathbf{C}_{\mathrm{clb}}} \mathbf{f}_{\mathbf{C}_{\mathbf{b}}} \\
\mathbf{v}_{\mathbf{C}_{\mathrm{c} 2}} & =\mathbf{Y}_{\mathbf{C}_{\mathrm{c} 2 \mathrm{~b}}} \mathbf{f}_{\mathbf{C}_{\mathrm{b}}} .
\end{aligned}
$$

It was shown by Bobrovnitskii [26] that for a coupled assembly the resultant velocity field in a receiver sub-structure, due to an applied force on a source sub-structure, may be reproduced identically through the application of the negative blocked force vector at the coupling interface. With this equivalent field representation in mind, equations A.1-A.3 may be rewritten as,

$$
\begin{aligned}
\mathbf{v}_{\mathbf{C}_{\mathrm{a}}} & =-\mathbf{Y}_{\mathbf{C}_{\mathrm{ac} 2}} \overline{\mathbf{f}}_{\mathbf{R}_{\mathrm{c} 2}} \\
\mathbf{v}_{\mathbf{C}_{\mathrm{c} 1}} & =-\mathbf{Y}_{\mathbf{C}_{\mathrm{c} 122}} \overline{\mathbf{f}}_{\mathbf{R}_{\mathrm{c} 2}} \\
\mathbf{v}_{\mathbf{C}_{\mathrm{c} 2}} & =-\mathbf{Y}_{\mathbf{C}_{\mathrm{c} 2 \mathrm{c} 2}} \overline{\mathbf{f}}_{\mathbf{R}_{\mathrm{c} 2}}
\end{aligned}
$$

where $\overline{\mathbf{f}}_{\mathbf{R}_{\mathrm{c} 2}}$ is the blocked force at interface $c_{2}$, due to the applied force at $b$. Equating equations A.1 and A.2, whilst eliminating $\mathbf{f}_{\mathbf{C}_{\mathbf{b}}}$, allows the following equality to be established,

$$
\mathbf{Y}_{\mathbf{C}_{\mathrm{ab}}}^{-1} \mathbf{v}_{\mathbf{C}_{\mathrm{a}}}=\mathbf{Y}_{\mathbf{C}_{\mathrm{clb}}}^{-1} \mathbf{v}_{\mathbf{C}_{\mathrm{cl}}}
$$

Substitution of equations A.4 and A.5 into A.7 yields,

$$
\mathbf{Y}_{\mathbf{C}_{\mathrm{ab}}}^{-1} \mathbf{Y}_{\mathbf{C}_{\mathrm{ac} 2}} \overline{\mathbf{f}}_{\mathbf{R}_{\mathrm{c} 2}}=\mathbf{Y}_{\mathbf{C}_{\mathrm{clb}}}^{-1} \mathbf{Y}_{\mathbf{C}_{\mathrm{clc} 2}} \overline{\mathbf{f}}_{\mathbf{R}_{\mathrm{c} 2}}
$$

Let us now consider the application of multiple forces at $b, \mathbf{f}_{\mathbf{C}_{\mathbf{b}_{\mathbf{i}}}}$. Each of these applied forces will result in a blocked force at the interface $c_{2}, \overline{\mathbf{f}}_{\mathbf{R}_{\mathbf{c}_{2}}}$. These blocked forces may be arranged as columns of a blocked force matrix, $\overline{\mathbf{F}}_{\mathbf{R}_{\mathbf{c} 2}}$. With free reign over the nature of the applied forces, we are able to ensure the invertability of this matrix. We are therefore 
able to eliminate the blocked force terms by first constructing the blocked forces matrix $\overline{\mathbf{f}}_{\mathbf{R}_{\mathrm{c} 2}} \rightarrow \overline{\mathbf{F}}_{\mathbf{R}_{\mathrm{c} 2}}$, and subsequently post-multiplying both sides of equation A.8 by its inverse, $\overline{\mathbf{F}}_{\mathbf{R}_{\mathrm{c} 2}}^{-1}$.

$$
\mathbf{Y}_{\mathrm{C}_{\mathrm{ab}}}^{-1} \mathbf{Y}_{\mathrm{C}_{\mathrm{ac} 2}}=\mathbf{Y}_{\mathrm{C}_{\mathrm{clb}}}^{-1} \mathbf{Y}_{\mathrm{C}_{\mathrm{clc} 2}}
$$

The pre-multiplication of equation A.9 by $\mathbf{Y}_{\mathbf{C}_{\text {clb }}}$, followed by the reciprocal substitution, $\mathbf{Y}_{\mathbf{C}_{\text {ac } 2}}=\mathbf{Y}_{\mathbf{C}_{\mathrm{c} 2 \mathrm{a}}}^{\mathbf{T}}$, yields the round trip identity for a dual contact interface,

$$
\mathbf{Y}_{\mathbf{C}_{\mathrm{clc} 2}}=\mathbf{Y}_{\mathbf{C}_{\mathrm{clb}}} \mathbf{Y}_{\mathrm{Cab}_{\mathrm{ab}}}^{-1} \mathbf{Y}_{\mathrm{C}_{\mathrm{c} 2 \mathrm{a}}}^{\mathrm{T}}
$$

or by reciprocity,

$$
\mathbf{Y}_{\mathbf{C}_{\mathrm{c} 2 \mathrm{c} 1}}=\mathbf{Y}_{\mathrm{C}_{\mathrm{c} 2 \mathrm{a}}} \mathbf{Y}_{\mathbf{C}_{\mathrm{ba}}}^{-1} \mathbf{Y}_{\mathbf{C}_{\mathrm{clb}}}^{\mathbf{T}} \text {. }
$$

The above relation allows for the transfer mobility $\mathbf{Y}_{\mathbf{C}_{\mathrm{c} c \mathrm{c}}}$ to be calculated without having to excite either interface. Instead, excitations are relocated to the remote DoFs $a$ and $b$.

The collocation of $c_{1}$ and $c_{2}$ such that $c_{1}=c_{2}=c$, can be seen to reduce equation A.10 to the standard round trip identity for the point mobility, as presented in [23, 27].

\section{References}

[1] A.T Moorhouse. Virtual acoustic prototypes: Listening to machines that don't exist. In Acoustics Australia, volume 33, pages 97-105, 2005.

[2] J.W.R. Meggitt, A.S. Elliott, and A.T. Moorhouse. Virtual assemblies and their use in the prediction of vibro-acoustic responses. In Proceedings of the Institute of Acoustics, Warickshire, 2016.

[3] D.D. Klerk, D.J. Rixen, and S.N. Voormeeren. General Framework for Dynamic Substructuring: History, Review and Classification of Techniques. AIAA Journal, 46(5):1169-1181, may 2008.

[4] K.G. Mcconnell. The Elusive Free-Free Boundary Condition by. Conference: 2006 IMAC-XXIV: Conference and Exposition on Structural Dynamics, page 6, 2006.

[5] C. Höller and B.M. Gibbs. Indirect determination of the mobility of structure-borne sound sources. Journal of Sound and Vibration, 344:38$58,2015$.

[6] S.N. Voormeeren and D.J. Rixen. A family of substructure decoupling techniques based on a dual assembly approach. Mechanical Systems and Signal Processing, 27:379-396, feb 2012.

[7] W. D'Ambrogio and A. Fregolent. The role of interface DoFs in decoupling of substructures based on the dual domain decomposition. Mechanical Systems and Signal Processing, 24(7):2035-2048, oct 2010.

[8] J. Zhen, T.C. Lim, and G. Lu. Determination of system vibratory response characteristics applying a spectral-based inverse sub-structuring approach. Part 1: analytical formulation. International Journal of Vehicle Noise and Vibration, 1(1), 2004.

[9] J. Zhen, T.C. Lim, and G. Lu. Determination of system vibratory response characteristics applying a spectral-based inverse sub-structuring approach. Part II: motor vehicle structures. International Journal of Vehicle Noise and Vibration, 1(1), 2004.

[10] G. Pavić and A.S. Elliott. Structure-Borne Sound Characterization of Coupled Structures - Part I : Simple Demonstrator Model. Journal of Vibration and Acoustics, 132(August):1-7, 2010.

[11] G. Pavić and A.S. Elliott. Structure-Borne Sound Characterization of Coupled Structures - Part II : Feasibility Study. Journal of Vibration and Acoustics, 132(August):1-13, 2010.

[12] J. Wang, G. Sun, L. Lu, and Q. Wang. General Formulation of Inverse Substructuring Method for Multicoordinate Coupled System. Advances in Mechanical Engineering, 2014:1-7, 2014

[13] L. Keersmaekers, L. Mertens, R. Penne, P. Guillaume, and G. Steenackers. Decoupling of mechanical systems based on in-situ frequency response functions: The link-preserving, decoupling method. Mechanical Systems and Signal Processing, 58-59:340-354, 2015.

[14] X. Liao, S. Li, L. Liao, and H. Meng. Virtual decoupling method: a novel method to obtain the FRFs of subsystems. Archive of Applied Mechanics, 2017.

[15] Z. Wang, P. Zhu, and Z. Liu. Relationships between the decoupled and coupled transfer functions: Theoretical studies and experimental validation. Mechanical Systems and Signal Processing, 98:936-950, 2018.

[16] J.W. Verheij. Multi-path sound transfer from resiliently mounted shipboard machinery. PhD thesis, 1982.

[17] International Organization for Standardization. BS EN ISO 10846-1:2008 Acoustics and vibration - Laboratory measurement of vibroacoustic transfer properties of resilient elements, Part 1: Principles and guidelines, 2008.

[18] J.W.R. Meggitt, A.S. Elliott, and A.T. Moorhouse. In-situ determination of dynamic stiffness for resilient elements. Journal of Mechanical Engineering Science, 230(6):986-993, 2015.

[19] D.J. Rixen, A. Boogaard, M.V. van der Seijs, G. van Schothorst, and T. van der Poel. Vibration source description in substructuring: A theoretical depiction. Mechanical Systems and Signal Processing, 60-61:498-511, 2015.

[20] D.J. Ewins. Modal Testing Theory and Practice, 1986.

[21] J.W.R. Meggitt. On In-situ Methodologies for the Characterisation and Simulation of Vibro-Acoustic Assemblies. Phd, University of Salford, 2017.

[22] M.V. van der Seijs, D. de Klerk, and D.J. Rixen. General framework for transfer path analysis: History, theory and classification of techniques. Mechanical Systems and Signal Processing, pages 1-28, 2015. 
[23] A.T. Moorhouse, T.A. Evans, and A.S. Elliott. Some relationships for coupled structures and their application to measurement of structural dynamic properties in situ. Mechanical Systems and Signal Processing, 25(5):1574-1584, jul 2011.

[24] International Organization for Standardization. BS ISO 9611:1996 Acoustics - Characterization of sources of structure-borne sound with respect to sound radiation from connected structures - Measurement of velocity at the contact points of machinery when resiliently mounted, 1996.

[25] A.T. Moorhouse. Virtual acoustic prototypes as a tool for low-noise design. In Proceedings of the Institute of Acoustics, volume 24, 2002.

[26] Y.I. Bobrovnitskii. A theorem on the representation of the field of forced vibrations of a composite elastic system. Acoustical Physics, 47(5):586-589, 2001.

[27] A.T. Moorhouse and A.S. Elliott. The "round trip" theory for reconstruction of Green's functions at passive locations. The Journal of the Acoustical Society of America, 134(5):3605-3612, nov 2013. 